九州大学学術情報リポジトリ

Kyushu University Institutional Repository

\title{
Interactive Evolutionary Computation for Analyzing Human Characteristics
}

Takagi, Hideyuki

Faculty of Design, Kyushu University

http://hdl. handle. net/2324/1434435

出版情報: Symposium on Emergent Trends in Artificial Intelligence \& Robotics (SETINAIR), pp.189-195, 2013-09. Center for Intelligent Technologies

バージョン :

権利関係 : 


\title{
Interactive Evolutionary Computation for Analyzing Human Characteristics
}

\author{
Hideyuki Takagi
}

\begin{abstract}
We emphasize that interactive evolutionary computation (IEC) can be used not only to optimize a target system based on an IEC user's subjective evaluations but also to analyze the characteristics of the IEC user. We introduce four research works as concrete examples of this new research direction: measuring a perceived range for emotional expressions, finding unknown auditory knowledge through hearing-aid fitting and cochlear implant fitting, and modeling of human awareness mechanism.
\end{abstract}

\section{Introduction}

Interactive evolutionary computation (IEC) is a framework for optimizing a target system based on human subjective evaluations. There are many tasks which performances are hard to be measured or almost impossible but can be evaluated by human beings. IEC shown in Fig. 1 (a) can optimize such tasks by involving a human user in an optimization loop.

The first direction of IEC research is to expand IEC application areas. IEC has been applied to wide variety of areas. They are roughly categorized into three: (1) artistic applications such as creating computer graphics (CG), music, editorial design, and industrial design, (2) engineering applications such as acoustic or image processing, robotics control, data mining, generating programming code, and media database retrieval, and (3) others such as educations, games, and geological simulation. See these perspectives in [8].

The second direction of IEC research is to reduce IEC user fatigue and make IEC practical. IEC users must repeat evaluations many times and feedback them to

Hideyuki Takagi

Kyusyu University, Fukuoka, Japan, e-mail: http://www.design.kyushu-u.ac.jp/ takagi/ 
a tireless computer. This nature causes IEC user fatigue, and especially, it becomes a serious problem for practical use when end-users use IEC.

Accelerating IEC search is one of solutions for this fatigue problem, and developing new IEC framework with less human fatigue is other solution. We introduce our trials in this paper. Besides them, there are several other approaches to overcome the fatigue problem such as improving IEC user interface, allowing an IEC user to intervene in an EC search, introducing a user model made by machine learning, and others. See these works in [8].

The third direction of IEC research is to use the IEC as a tool to analyze human characteristics; see Fig. 1(b). This is a new and unique approach, and there are few related works so far. We focus on this third research direction in this paper.

Since an IEC target system is optimized based on a human psychological evaluation scale, we may know the scale indirectly by analyzing the optimized target system. It somehow has similarity to reverse engineering in software engineering. Other explanation of this approach is that IEC is a tool to visualize impressions or images in mind. Artists have skills for expressing them by drawing pictures, playing musical instruments, programming computer graphics and writing in poems, for example. However, it is hard for many ordinary people who have no such skills to express the impressions or mental images. IEC helps those who have less skill to express the mental images using IEC-based systems.

Thanks to this kind of IEC use, we may be able to analyze human characteristics by analyzing obtained optimized systems and their system outputs. Through the analysis, we are looking forward to finding out new psychological or physiological unknown facts.

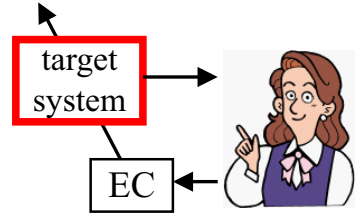

(a)

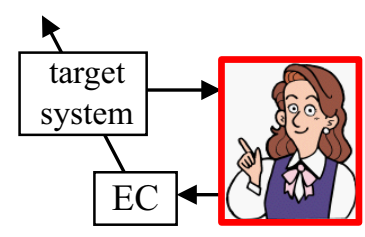

(b)

Fig. 1 IEC frameworks (a) for optimization and (b) for human science.

\section{Measuring a Perceived Range for Emotional Expressions}

We applied IEC to measure a happy-sad range in human mind and compared the ranges of schizophrenics and mental healthy people. Some therapists feel that face emotional impressions of schizophrenic patients are fewer than those of mental 
healthy people through their experiences. However, there was no way to measure the range.

We asked 3 schizophrenics and 5 mental healthy students to design 3-D CG lighting of the happy impression and the sad impression using our IEC-based 3-D CG lighting design support system [1,2] and asked 33 human subjects to evaluate 28 pairs $\left({ }_{(3+5)} \mathrm{C}_{2}\right)$ of designed lighting images. Fig. 2 is the psychological scale of happy constructed using the Scheffé's method of paired comparison.

The happy-sad ranges (Fig. 3) obtained from the experimental results imply that it is hard for schizophrenic patients to identify especially a happy impression in lighting. It is expected that this IEC approach may provide new data that are helpful for psychiatric diagnostics [10].

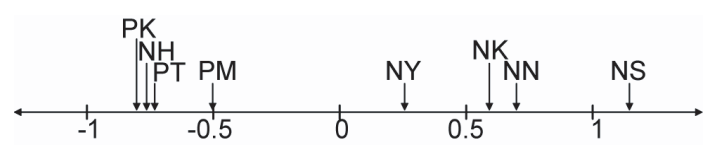

Fig. 2 Psychological scale constructed using the Scheffé's method of paired comparison and impression levels of the eight best lightings designed by three schizophrenics (PK, PT, and PM) and five mental healthy students (NH, NY, NK, NN, and ND). The bigger measure values, the higher evaluation of happy.

\section{Hearing-aid Fitting and Finding Unknown Knowledge}

IEC is the best way for hearing-aid fitting because sound qualities for a certain hearing-aid user cannot be measured. Other advantage is that it allows us to fit a hearing aid using any daily-life sounds, while conventional fitting method has to use only pure tones and narrow band noise. Thanks to this feature, we could compare fitting characteristics optimized using only pure sounds and daily-life sounds and find several unknown facts [11].

They are: the characteristics of hearing aids optimized using speech sounds were different from those optimized using pure tones or band pass noise; those optimized using speech sounds of speaker $i$ with/without noise were almost similar to those optimized using speech sounds of speaker $j$ with/without noise $(i \neq j)$; those optimized using speech sounds were different from those optimized using music.

Nobody had known the facts that the best characteristics of hearing-aids depend on sound types used for fitting. It implies that an audible range in human sense level is not the final cue for the best hearing. We could find these observations thanks to an IEC technique.

From the obtained facts, we can imagine that the ideal hearing-aids in the future would have multiple best hearing-aid characteristics for different acoustic environments and switch their characteristics based on the change of the environments. 


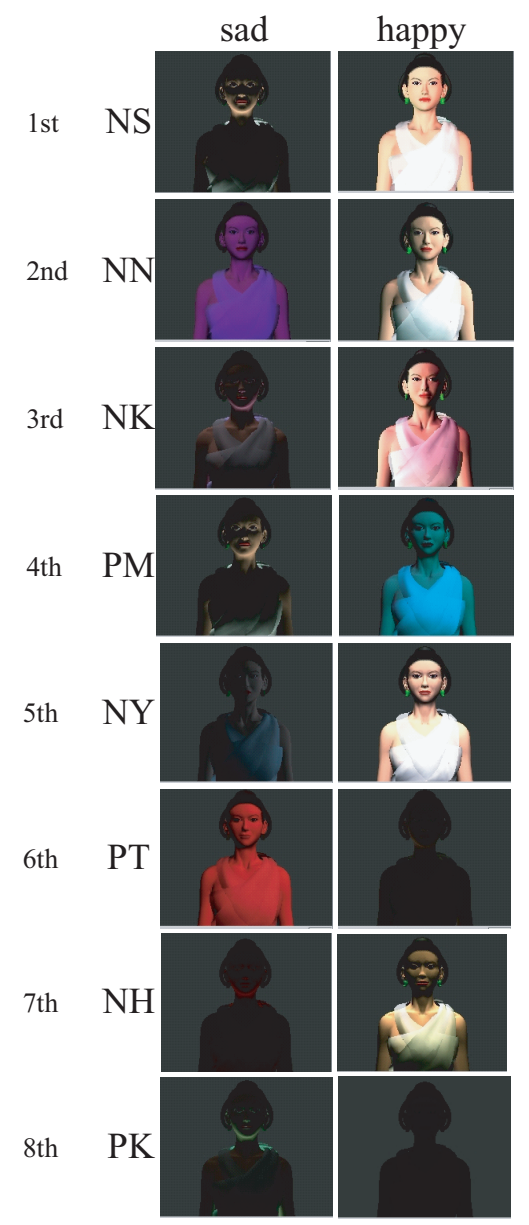

Fig. 3 Rank order of happy-sad expression range obtained as a difference between two happy and sad scales. See the subject ID's in the caption of Fig. 2.

\section{Cochlea Implant Fitting and Finding Unknown Knowledge}

Cochlea-implant fitting is a similar task with hearing-aid fitting and has been conducted based on two hypotheses for better fitting: (1) the more electric channels of a cochlea-implant, the better and (2) the wider dynamic range of each channel, the better. As frequency resolution increases according to the number of electric channels, the hypothesis (1) means that higher frequency resolution helps to distinguish the difference of frequency characteristics of phonemes; this hypothesis sounds natural. The hypothesis (2) means that enabling a user to hearing sounds from the minimum level to the maximum comfortable level is helpful to distinguish sounds; this hypothesis also sounds natural. 


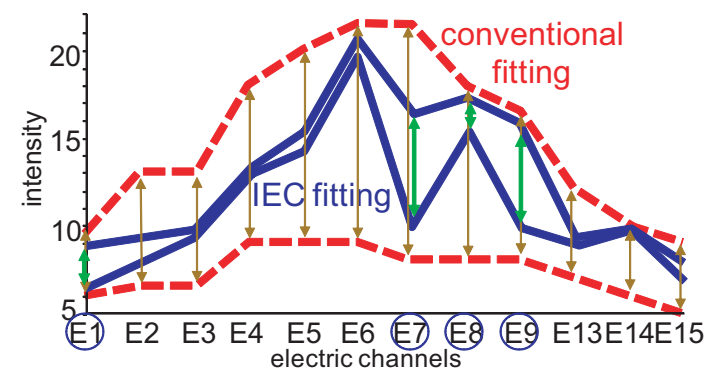

Fig. 4 Fitting characteristics of conventional cochlear implant fitting and that used IEC. Horizontal axis means electric channels and a vertical axis means electric voltage of each channel. This figure was remade based on an image in [6].

Interactive genetic algorithms (IGA) was used to tune the fitting parameters of cochlea implants [6]. Their experimental result was that dynamic ranges of all 15 channels were almost 0 except 3 or 4 channels, and the dynamic ranges of the exceptional 3 or 4 channels were narrower than the maximum ranges (see Fig. 4). Nevertheless, its recognition rate with IGA fitting was higher than that of manual fitting.

This result did not match to the mentioned two hypotheses. It implies that there must be unknown audio-psychophysiological facts. We are conducting this cochlea-implant fitting using paired comparison-based interactive differential evolution (IDE) [12] together with medical departments of two universities and trying to find the unknown facts.

\section{IEC for Awareness Science}

Although there are many application papers on awareness computing, such as content awareness, location awareness, power awareness, and others, there are few papers that focus on basic research for awareness science such as analyzing awareness mechanisms and making awareness models. Once computer has an awareness model, it must be useful for communication with human users and support human awareness. IEC can be a tool for this research.

There must be several types of human awareness at different psychological levels including a sensory level, a perceptual level, and a cognitive level. To start this basic research, let us define awareness as realize latent variables that explain the relationship between inputs to a human and outputs from the human.

Let us consider an example case that someone finds a preferred pot but cannot explain why he or she prefers it. This case means that the relationship between a visual image of the pot (input $\mathbf{x}$ ) and the evaluation (output $z$ ) is complex. After thinking for a while, he or she may become aware that the vertical-horizontal ratio 
of the pot $(r)$ and the curvature of design pattern $(c)$ are the points underpinning their evaluation and become to be able to explain the reason for their preference as Fig. 5. Then, we may say that he or she is aware of the two hidden variables of the vertical-horizontal ration of the pot and the curvature of design pattern.

That is, we can say that " $f()$ for $z=f(x)$ was so complex that he orshe could not explain the input-output relationship at the first glance. However, he or she found the latent variables, $r$ and $c$, and could interpret them as $\mathbf{z}=f(\mathbf{x})=g_{1}(r, c)$, where $r=g_{2}(\mathbf{x})$ and $c=g_{3}(\mathbf{x})$." In other words, we can say that he or she became to explain a complex relationship between $\mathbf{z}$ and $\mathbf{x}$ explicitly thanks to $r$ and $c$.

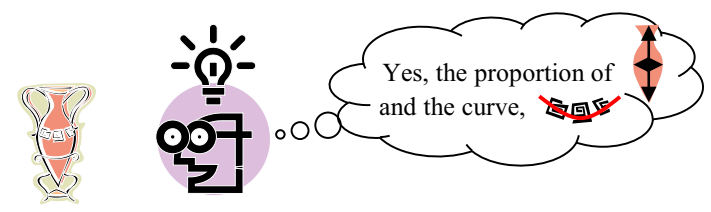

Fig. $5 \mathrm{He}$ or she is aware of the vertical-horizontal ratio of the pot $(r)$ and the curvature of design pattern $(c)$ as key points of his or her preference of the pot.

One approach to model an awareness mechanism is to make an IEC user model using machine learning (Fig. 6) and resolve the obtained user model (Fig. 7). Now we are considering some approaches for obtaining latent variables: (a) structure analysis of a structured NN-FS model, (b) introducing statistical methods for finding latent variables, (c) making a learning model by a math equation using genetic programming and analyzing the obtained equation, and (d) others.

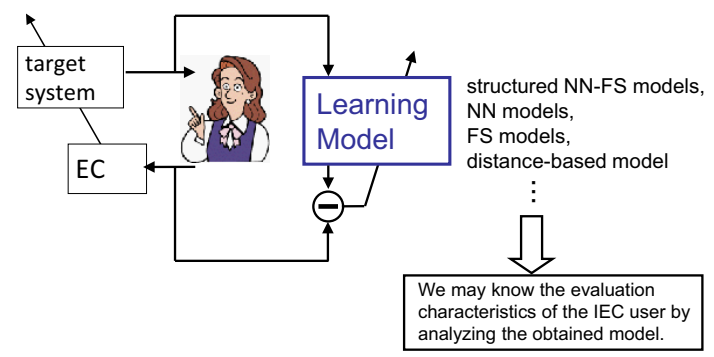

Fig. 6 Leaning inputs and outputs to/from an IEC user and making its user model. 


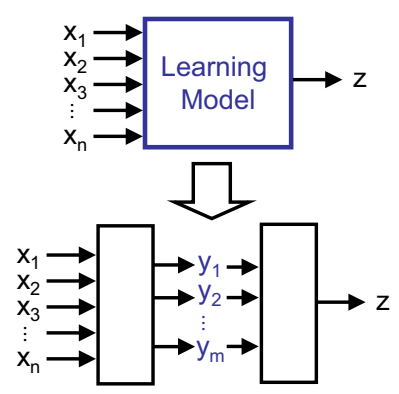

Fig. 7 resolving an IEC user model using latent variables.

\section{Conclusions}

IEC was started since Richard Dawkins demonstrated biomorph, evolving 2-D line figures, in 1986, and its research increased from 1990's. IEC applications were spread from CG applications and engineering, edutainment, and many other areas. Now the number of IEC papers became 835 (in Scopus database as of July, 2013).

Still now, majority of IEC research is on optimizing target systems. Although expanding IEC to application areas that are hard for conventional optimization methods are useful and important, IEC has other potentials. To demonstrate it, we introduced unique our IEC research, i.e. IEC for human science, as the third direction of IEC research. We hope that many people realize that the capability of IEC is not only optimization from these efforts and IEC can contribute quite widely.

\section{Acknowledgment}

This work was supported in part by Grant-in-Aid for Scientific Research (23500279).

\section{References}

1. Aoki, K. and Takagi, H., "3-D CG lighting with an interactive GA," 1st Int. Conf. on Conventional and Knowledge-based Intelligent Electronic Systems (KES'97), pp.296-301, Adelaide, Australia (May, 1997).

2. Aoki, K. and Takagi, H., "Interactive GA-based design support system for lighting design in 3D computer graphics," Trans. of IEICE, vol.J81-DII, no.7, pp.1601-1608 (1998) (in Japanese).

3. Dawkins, Richard, The blind watchmaker, W. W. Norton \& Company, Inc., New York, USA, (Dec., 1986).

4. Ingu, T. and Takagi, H., "Accelerating a GA convergence by fitting a single-peak function," IEEE Int. Conf. on Fuzzy Systems (FUZZ-IEEE'99), Seoul, Korea, pp.1415-1420 (Aug., 1999). 
5. Jin, YaoChu, "A comprehensive survey of fitness approximation in evolutionary computation," Soft Computing, vol.9, no.1, pp.3-12 (2005).

6. Legrand, P., Bourgeois-Republique, C., Péan, V., et al., "Interactive evolution for cochlear implants fitting," Genetic Programming and Evolvable Machines, vol.8, no.4, pp.319-354, 2007.

7. Pei, P. and Takagi, H., "Fourier analysis of the fitness landscape for evolutionary search acceleration," IEEE Congress on Evolutionary Computation (CEC2012), pp.1-7, Brisbane, Australia (June, 2012).

8. Takagi, Hideyuki, "Interactive evolutionary computation: fusion of the capabilities of EC optimization and human evaluation," Proceedings of the IEEE, vol.89, no.9, pp.1275-1296 (2001).

9. Takagi, H., Ingu, T., and Ohnishi, K., "Accelerating a GA convergence by fitting a singlepeak function," J. of Japan Society for Fuzzy Theory and Intelligent Informatics, vol.15, no.2, pp.219-229 (2003) (in Japanese).

10. Takagi, H., Takahashi, T., and Aoki, K., "Applicability of interactive evolutionary computation to mental health measurement," IEEE Int. Conf. on Systems, Man, and Cybernetics (SMC2004), the Hague, the Netherlands, pp.5714-5718 (Oct., 2004).

11. Takagi, H. and Ohsaki, M., "Interactive evolutionary computation-based hearing-aid fitting," IEEE Trans. on Evolutionary Computation, vol.11, no.3, pp.414-427 (2007).

12. Takagi, H. and Pallez, D., "Paired comparison-based interactive differential evolution," 1st World Congress on Nature and Biologically Inspired Computing (NaBIC2009), Coimbatore, India, pp.375-480 (Dec., 2009). 